\section{Southern Blotting and Related DNA Detection Techniques}

Terence A Brown, University of Manchester Institute of Science and Technology, Manchester, UK

Southern blotting is a technique for transfer of DNA molecules from an electrophoresis gel to a nitrocellulose or nylon membrane, and is carried out prior to detection of specific molecules by hybridization probing.

\section{Secondary article}

Article Contents
- Introduction
- The Principle of the Southern Blot
- Preparation of DNA Prior to Southern Blotting
- DNA Blotting Techniques
- DNA/DNA Hybridization
- Types of Hybridization Probes
- Applications and Limitations of Southern Blotting
- Summary

\section{Introduction}

Southern blotting is one of the central techniques in molecular biology. First devised by E. M. Southern (1975), Southern blotting results in transfer of DNA molecules, usually restriction fragments, from an electrophoresis gel to a nitrocellulose or nylon sheet (referred to as a 'membrane'), in such a way that the DNA banding pattern present in the gel is reproduced on the membrane. During transfer or as a result of subsequent treatment, the DNA becomes immobilized on the membrane and can be used as a substrate for hybridization analysis with labelled DNA or RNA probes that specifically target individual restriction fragments in the blotted DNA. In essence, Southern blotting is therefore a method for 'detection of a specific restriction fragment against a background of many other restriction fragments' (Brown, 1999). The restricted DNA might be a plasmid or bacteriophage clone, Southern blotting being used to confirm the identity of a cloned fragment or to identify an interesting subfragment from within the cloned DNA, or it might be genomic DNA, in which case Southern blotting is a prelude to techniques such as restriction fragment length polymorphism (RFLP) analysis.

In this article the general principles of Southern blotting are described, followed by overviews of the methodologies used for preparation of DNA prior to blotting, for blotting itself, and for hybridization analysis. The article concludes with a survey of the applications and limitations of Southern blotting.

\section{The Principle of the Southern Blot}

The ability of nitrocellulose powder or sheets to bind DNA has been known for many years and was utilized in the 1950s and 1960s in various types of nucleic acid hybridization studies. In these early techniques the immobilized DNA was unfractionated, simply consisting of total DNA that was bound to nitrocellulose powder or spotted onto a nitrocellulose sheet. The introduction in the early 1970s of gel electrophoresis methods that enable restriction fragments of DNA to be separated on the basis of their size prompted the development of techniques for the transfer of separated fragments en masse from gel to nitrocellulose support. The procedure described by Southern (1975), involving capillary transfer of DNA from the gel to a nitrocellulose sheet placed on top of it, was simple and effective, and although embellished over the years this original procedure differs only slightly from the routine method still used in many molecular biology laboratories.

\section{The original methodology for Southern blotting}

The original methodology for Southern blotting is illustrated in Figure 1. An agarose electrophoresis gel, containing the fractionated restriction fragments, is placed on a filter paper wick that forms a connection between the gel and a reservoir of high-salt buffer. The nitrocellulose

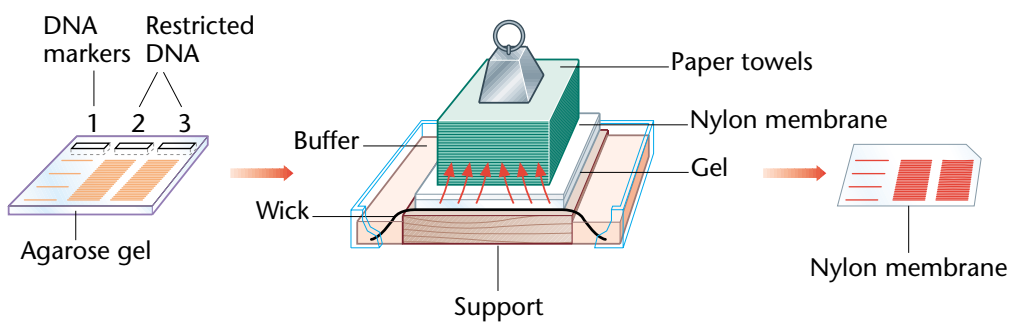

Figure 1 Southern blotting. Reproduced from Brown (1999) with permission of BIOS Scientific Publishers Ltd, Oxford. 
membrane is placed on top of the gel and covered with a tower of paper towels that are held in place with a weight. Capillary action results in the buffer soaking through the filter paper wick, gel and membrane and into the paper towels. As the buffer passes through the gel the DNA fragments are carried with it into the membrane, where they become bound to the nitrocellulose. Effective transfer of fragments up to $15 \mathrm{~kb}$ in length takes approximately $18 \mathrm{~h}$, roughly equivalent to 'overnight'. The only technical complication is the possibility that the buffer bypasses the gel by soaking directly from wick to paper towels, which is unlikely if the setup is assembled carefully.

\section{More recent modifications to Southern blotting}

The system shown in Figure 1 is an accurate description of Southern blotting as still carried out in many laboratories, but various modifications have been introduced over the years to improve the efficiency of DNA transfer from gel to membrane. The major improvement has been the introduction of nylon membranes, which have three advantages over their nitrocellulose counterparts. First, nylon membranes are less fragile than nitrocellulose sheets, the latter tending to crack if handled roughly during Southern blotting, and usually disintegrating if attempts are made to carry out more than two or three hybridization analyses with the same blot. Nylon membranes cannot be damaged by handling and a single blot can be rehybridized up to ten times, this limit being due not to eventual breakage of the membrane but to the gradual loss of the blotted DNA during repeated hybridizations. The second advantage of nylon membranes is that under certain conditions (a positively charged membrane and an alkaline transfer buffer) the transferred DNA becomes covalently bound to the membrane during the transfer process. This is not the case with a nitrocellulose membrane, which initially binds DNA in a semipermanent manner, immobilization occurring only when the membrane is baked at $80^{\circ} \mathrm{C}$. Transfer onto a positively charged nylon membrane can therefore reduce the possible loss of DNA that might occur by leaching through the membrane during the blotting process; it is also quicker, the transfer time being reduced from $18 \mathrm{~h}$ to $2 \mathrm{~h}$. Finally, nylon membranes efficiently bind DNA fragments down to $50 \mathrm{bp}$ in length, whereas nitrocellulose membranes are effective only with molecules longer than $500 \mathrm{bp}$. Nitrocellulose has not, however, been completely superseded because it has one significant advantage compared with nylon membranes: a reduced amount of background hybridization, especially with probes that have been labelled with nonradioactive markers.

Other changes to the original Southern blotting procedure have been introduced to speed up and improve the efficiency of the transfer. Changes to the architecture of the capillary blotting system have been proposed but these alternative arrangements are more complicated to set up than the simple upward transfer shown in Figure 1, and none provides any major improvement in speed or efficiency. Improvements can, however, be achieved by the use of a noncapillary transfer method, such as electroblotting, which employs electrophoresis to move the DNA from the gel to the membrane. This technique was originally developed for blotting polyacrylamide gels, whose pore sizes are too small for effective capillary transfer of DNA, and has also been applied to agarose gels (Bittner et al., 1980). Electroblotting is more rapid than capillary transfer but has to be carried out with care because problems can arise with bubble formation if the gel gets too hot during the transfer. Vacuum blotting is a more popular choice for agarose gels, a vacuum pressure being used to draw the buffer through the gel and membrane more rapidly than occurs by simple capillary action, enabling the transfer time to be reduced to as little as $30 \mathrm{~min}$. Attempts have also been made to dispense entirely with Southern blotting and carry out hybridization analysis directly with the electrophoresis gel, immobilization of the DNA being achieved by drying the gel so that the DNA becomes trapped in the dehydrated gel matrix or bound to a solid support on to which the gel is placed prior to drying. These techniques have proved useful for some applications but suffer from high background signals after hybridization analysis.

\section{Preparation of DNA Prior to Southern Blotting}

\section{Preparation of genomic DNA}

The techniques used to prepare DNA for Southern blotting depend on the type of DNA that is being studied. For genomic DNA, the objective is to obtain molecules that have not become extensively fragmented by random shearing during the extraction process, so that specific restriction fragments of $20 \mathrm{~kb}$ and more can be obtained. Cells must therefore be broken open under relatively gentle conditions. For tissue culture cells and blood samples incubation in a buffer containing a detergent such as sodium dodecyl sulfate (SDS) is usually sufficient to disrupt the cell membranes and release high-molecular weight DNA. Bacteria, which are surrounded by a cell wall, can be gently broken open by treatment with a combination of lysozyme and ethylenediaminetetraacetate (EDTA). Lysozyme is an enzyme that degrades some of the polymeric compounds present in the bacterial cell wall, and EDTA chelates magnesium ions that are required for integrity of the polymeric structure. Subsequent addition of a detergent causes the cells to burst. Other microorganisms with tough cell walls, such as yeast and fungi, can also 
be treated with appropriate cell wall-degrading enzymes, and the same is true for plant cells though the latter are usually broken open by physical grinding of tissues that have been frozen in liquid nitrogen. This causes some fragmentation of the DNA but provides a much higher yield.

After cell disruption, genomic DNA extraction procedures continue with steps aimed at removing the major biochemicals other than DNA present in the initial extract. A protease such as proteinase $\mathrm{K}$ might be included in the buffer used for cell disruption, in order to begin the degradation of proteins in the extract, but deproteinization is routinely carried out by phenol extraction, the addition of phenol or a 1:1 mixture of phenol and chloroform resulting in precipitation of proteins. After centrifugation, the precipitated proteins migrate to the interface between the organic and aqueous phases, whereas the nucleic acids remain in the aqueous phase. For plant extracts, which contain greater quantities of carbohydrates than are found in most animal and microbial cells, an additional purification involving the detergent cetyltrimethylammonium bromide (CTAB) is frequently used. This compound specifically binds nucleic acids, improving their recovery during phenol extraction. Most extraction procedures also include digestion of RNA with ribonuclease and a final treatment with ethanol, which precipitates the remaining nucleic acid polymers, enabling ribonucleotides and other low-molecular weight contaminants to be removed. The precipitated DNA is dried and then redissolved in water or a Tris-EDTA buffer.

\section{Preparation of plasmid or bacteriophage DNA}

Special techniques must be used if the objective is to obtain plasmid or bacteriophage DNA from bacterial clones. Plasmid DNA can be obtained by any one of several techniques that exploit the physical differences between plasmids and bacterial genomic DNA, plasmids being small supercoiled molecules whereas genomic DNA, after cell disruption, is present as long, linear fragments. A popular method involves treatment of the cell extract with alkali, which precipitates the linear DNA but leaves supercoiled plasmids in solution. Bacteriophage DNA, for example recombinant $\lambda$ or M13 vectors, is usually obtained directly from bacteriophage particles secreted by infected bacteria, the purification process being simplified by the fact that these particles consist solely of DNA and protein.

\section{DNA Blotting Techniques}

After the purified DNA has been treated with one or more restriction endonucleases it is fractionated by agarose gel electrophoresis and the gel then pretreated prior to setting up the Southern blot. The pretreatment has two objectives. First, it is desirable to break the DNA molecules in individual bands within the gel into smaller fragments, because smaller fragments transfer more quickly than larger ones. This is achieved by soaking the gel in $0.25 \mathrm{~mol} \mathrm{~L}^{-1} \mathrm{HCl}$ for $30 \mathrm{~min}$, which results in a small amount of depurination - cleavage of the $\beta$ - $N$-glycosidic bond between purine bases (adenine or guanine) and the sugar component of their nucleotides - which is followed by decomposition of the sugar structure and breakage of the polynucleotide chain. The second pretreatment is with an alkaline solution that denatures the double-stranded DNA molecules by breakage of their hydrogen bonds, so the molecules become single-stranded. This aids their transfer and subsequent binding to the membrane, and also ensures that after binding the base-pairing components of the polynucleotides are available for hybridization with the probe.

If a nitrocellulose membrane is being used then the alkali pretreatment is followed by neutralization of the gel by soaking in a Tris-salt buffer, this step being essential because DNA does not bind to nitrocellulose at a $\mathrm{pH}$ of greater than 9.0. The Southern blot is then set up, as illustrated in Figure 1, with a high-salt transfer buffer, usually the formulation called ' $20 \times$ SSC', which comprises $3.0 \mathrm{~mol} \mathrm{~L}^{-1} \mathrm{NaCl}$ (salt) and $0.3 \mathrm{~mol} \mathrm{~L}^{-1}$ sodium citrate. The same buffer can be used for transfer to a nylon membrane, but with a positively charged nylon membrane an alkaline transfer buffer $\left(0.4 \mathrm{~mol} \mathrm{~L}^{-1} \mathrm{NaOH}\right)$ is used because, as described earlier, this results in immediate covalent attachment of the transferred DNA to the membrane. With this type of transfer the alkali pretreatment is unnecessary. The blot is then left for at least $18 \mathrm{~h}$ for a high-salt transfer, or $2 \mathrm{~h}$ for an alkaline blot.

After blotting, the transfer setup is dismantled and the membrane rinsed in $2 \times \mathrm{SSC}$ and left to dry. If the blot has been made onto a nitrocellulose or uncharged nylon membrane, then the DNA is only loosely bound to the membrane at this stage. More permanent immobilization must therefore be carried out, either by baking at $80^{\circ} \mathrm{C}$ for $2 \mathrm{~h}$, which results in noncovalent but semipermanent attachment of DNA to a nitrocellulose membrane, or UV irradiation, which results in covalent attachment of DNA to a nylon membrane.

\section{DNA/DNA Hybridization}

Hybridization analysis is based on the principle that two polynucleotides will form a stable hybrid by base-pairing if their nucleotide sequences are wholly or partly complementary. A specific restriction fragment in a Southern blot can therefore be detected if the membrane is probed with a second, labelled DNA molecule that has the same, or similar, sequence as the fragment being sought. We will 
examine the types of hybridization probe that can be used later; first we will survey the methodology used for hybridization analysis.

\section{Prehybridization of a Southern blot}

Hybridization analysis is carried out by soaking the Southern blot in a buffer containing the hybridization probe, usually in a tube that is constantly rotated so all parts of the membrane are exposed to the probe, or alternatively in a sealed plastic bag that is placed on a shaker. Hybridization is carried out in two stages. First, the membrane is prehybridized in a solution designed to block the unused DNA binding sites on the membrane surface. If this step is omitted then the probe will bind nonspecifically to the surface of the membrane and the signal resulting from hybridization to the specific restriction fragment will be difficult if not impossible to identify. The prehybridization solution therefore contains nonbiological polymeric compounds such as polyvinylpyrrolidone and/or biological polymers such as Ficoll (a carbohydrate-based compound), bovine serum albumin or dried milk. DNA from an organism unrelated to the one whose DNA has been blotted can also be used (salmon sperm DNA is a popular choice). Prehybridization takes between $15 \mathrm{~min}$ and $3 \mathrm{~h}$ at $68^{\circ} \mathrm{C}$, depending on the type of membrane.

\section{The hybridization and washing steps}

The second stage is the actual hybridization, which is carried out in a high-salt buffer containing a detergent, usually $2 \times \mathrm{SSC}+1 \% \mathrm{SDS}$. Two issues are critical at this stage of the experiment. First, enough probe DNA must hybridize to the target restriction fragment to produce a clear signal that can be discerned by the detection system appropriate for the label carried by the probe. For the most demanding applicationsuch as detection of a single copy gene in human genomic DNA, achieving sufficient sensitivity might be a problem even if the maximum amount of genomic DNA is loaded on to the electrophoresis gel (in practice, about $10 \mu \mathrm{g}$ of DNA per lane) and the probe has been labelled to its maximal specific activity ( $>10^{9} \mathrm{dpm} \mu \mathrm{g}^{-1}$ for a ${ }^{32} \mathrm{P}$ radioactive label). Increasingly greater problems will be encountered if a radioactive label other than ${ }^{32} \mathrm{P}$ is used, such as ${ }^{35} \mathrm{~S}$, which is generally suitable only for probing less complex DNAs such as restricted plasmid or bacteriophage clones, or if a nonradioactive probe is employed. Under these circumstances some increase in sensitivity can be obtained by including an inert polymer such as $10 \%$ dextran sulfate or $8 \%$ polyethylene glycol 6000 in the hybridization solution. These polymers are thought to induce the probe molecules to form networks so that greater amounts of DNA hybridize to the target sites on the membrane. In practice, a 10 - to 100 -fold increase in sensitivity can be achieved (Amasino, 1986).

The second critical factor that must be considered during the hybridization step is the specificity of the reaction. If the probe DNA has been carefully chosen then it will contain a region that is completely complementary to all or a part of the blotted restriction fragment that is being sought. If this hybridizing region in the probe is not completely complementary to the target, then it will at least have a region of strong similarity so that a stable hybrid can form. The problem is that the probe also has the potential to hybridize to any other blotted DNA fragments with which it has partial complementarity. The hybridization experiment will never give an unambiguous signal if the probe is more similar to a second restriction fragment than it is to the one being sought, but problems with nonspecific hybridization can still arise even if the best match is with the specific target. This means that the hybridization step must be carried out at a 'stringency' that results in the specific probe-target hybrid remaining stable while all other hybrids are unstable, the stringency being determined by the composition of the hybridization buffer and the temperature at which the experiment is carried out. Buffer composition is relevant because hybrid stability is dependent on the ionic strength and the presence of destabilizing agents, such as formamide, which disrupt hydrogen bonds. Temperature is relevant because the melting temperature ( $T_{\mathrm{m}}$, the highest temperature at which the hybrid is stable) of a fully base-paired hybrid is higher than that for one in which some base pairs have not formed because the probe and target DNAs are not fully complementary. Formation of the desired hybrid, and destabilization of nonspecific hybrids, can therefore be achieved by utilizing an appropriate combination of buffer composition and hybridization temperature. A number of different strategies are possible. If the probe is $>100 \mathrm{bp}$ in length, for example a cloned restriction fragment, then the initial hybridization is usually carried out at $68^{\circ} \mathrm{C}$ in a high-salt buffer, this representing highly stringent conditions under which only stable hybrids are expected to form, with very little if any nonspecific hybridization. With this strategy, washing steps that are carried out after hybridization are designed simply to remove the nonhybridized probe. However, if a short oligonucleotide probe is used (15-25 nucleotides in length), then the hybridization step is usually carried out under conditions of low stringency (typically at a temperature several degrees below the calculated $T_{\mathrm{m}}$ for the desired hybrid), so that all potential hybrids, including nonspecific ones, are able to form. Specificity is then achieved by a series of washes at increasing temperatures so that, hopefully, only the desired hybrid remains at the end of the procedure.

After washing, the membrane is subjected to the detection procedure appropriate for the label that has been used, for example autoradiography for a radioactive label. Subsequent reprobing is possible if the membrane is 
'stripped' by washing in a high-temperature buffer containing alkali and detergent to destabilize the hybridized DNA. This procedure is never wholly satisfactory as it is difficult to avoid removing some of the blotted DNA at the same time, so even nylon membranes carrying covalently bound DNA can only be reprobed ten or so times.

\section{Types of Hybridization Probes}

Any DNA molecule that is complementary to the restriction fragment being sought can be used as the probe. Depending on the application (see below), the probe might itself be a restriction fragment, or it might be a DNA molecule obtained by the polymerase chain reaction (PCR), or a cDNA prepared by complementary DNA synthesis from an mRNA template. Synthetic oligonucleotides are also frequently used as hybridization probes, especially when discrimination between very similar target DNAs is required. This is because oligonucleotides of 1525 nucleotides in length form hybrids whose melting temperatures can be estimated from the formula:

$$
\begin{aligned}
& T_{\mathrm{m}}=(2 \times \text { number of } \mathrm{A} \text { and } \mathrm{T} \text { nucleotides }) \\
& +(4 \times \text { number of } \mathrm{G} \text { and } \mathrm{C} \text { nucleotides }){ }^{\circ} \mathrm{C}
\end{aligned}
$$

The $T_{\mathrm{m}}$ values for most $15-25 \mathrm{mer}$ oligonucleotides fall between 40 and $90^{\circ} \mathrm{C}$. A single nonbase-paired nucleotide position in the hybrid between the oligonucleotide probe and target DNA can reduce the $T_{\mathrm{m}}$ by $3-5^{\circ} \mathrm{C}$, so careful control of the posthybridization wash temperature can distinguish a target site that is completely complementary to the probe from one that differs at just a single nucleotide position, enabling highly specific probing to be carried out.

A second popular type of hybridization probe is one made of RNA rather than DNA. RNA probes have a number of advantages compared with their DNA counterparts, the most important of which in practical terms is the availability of methods for synthesis in the test tube of large amounts of an RNA probe from a DNA molecule that has been cloned adjacent to a promoter for a bacteriophage RNA polymerase. Starting with $1 \mu \mathrm{g}$ of cloned DNA, purified T7 RNA polymerase, for example, can make up to $30 \mu \mathrm{g}$ of RNA in $30 \mathrm{~min}$, this RNA being labelled with a specific activity of over $10^{9} \mathrm{dpm} \mu \mathrm{g}^{-1}$ if a ${ }^{32} \mathrm{P}$-labelled ribonucleotide is included in the reaction mixture. RNA probes also have the advantage of being single-stranded, which means that the effective probe concentration does not decline during the hybridization, as happens with a double-stranded DNA probe due to base-pairing of the two complementary probe molecules to one another.

\section{Applications and Limitations of Southern Blotting}

The applications of Southern blotting are diverse and are not easily summarized in a short article. Two examples will suffice to illustrate the range of research questions to which the technique can be applied.

\section{Southern blotting in clone identification}

The commonest applications of Southern blotting occur during projects aimed at identification and cloning of a specified gene. Southern blotting of genomic DNA is used to identify one or more restriction fragments that contain the gene being sought and, after cloning and tentative identification of the desired recombinant by colony or plaque hybridization probing, Southern blotting of restricted clone DNA is used to confirm the clone identification and possibly to locate a shorter restriction fragment, containing the sequence of interest, from within the cloned DNA. The latter application is important because the interesting gene sequence might be just a few $\mathrm{kb}$ in length but contained initially in a genomic clone of several hundred $\mathrm{kb}$ prepared with a high-capacity vector such as a bacterial or yeast artificial chromosome. For Southern blotting to be applicable to gene identification it is of course necessary to have a suitable hybridization probe, one that will detect specifically the one or more restriction fragments that contain the gene being sought. Often the gene will have already been cloned as a cDNA that can be used as a probe for identification of the genomic version of the gene. Alternatively, if the gene has been cloned from a related organism, one in which the gene sequence is likely to be similar to that in the organism now being studied, then heterologous probing can be used, in which some noncomplementarity between probe and target is tolerated during the hybridization step. This approach can be used, for example, to identify homologues of human genes in the genomes of other primates, and can even be used across broad species barriers, for example by using Drosophila genes as probes for related human sequences. Another possibility is to use the amino acid sequence of the protein coded by the desired gene to design a mixed oligonucleotide probe, one that consists of a variety of related sequences that together include all the combinations that would code for a short segment of the amino acid sequence. For example, the mixed oligonucleotide $5^{\prime}-\mathrm{AA}(\mathrm{C} / \mathrm{U}) \mathrm{GA}(\mathrm{A} /$ G)AUGUU(C/U)UGGUA(C/U)GG-3', which contains sixteen different sequences, covers all possibilities for the amino acid sequence asparagine-glutamine-methioninephenylalanine-tryptophan-tyrosine-glycine and would be expected to detect the DNA sequence coding for this protein segment when used at a sufficiently high stringency in a Southern hybridization experiment. 


\section{Restriction fragment length polymorphism analysis}

The second major application of Southern hybridization is in the technique called restriction fragment length polymorphism (RFLP) mapping, which is important in construction of genome maps (Brown, 1999). Treatment of genomic DNA from different individuals with a single restriction enzyme does not always give the same set of fragments because some restriction sites are polymorphic, being present in some individuals but absent in others, usually because a point change in the nucleotide sequence changes the restriction site into a sequence not recognized by the restriction enzyme. These polymorphic sites can be used as markers in genetic mapping and were of considerable importance in construction of the first comprehensive human maps (Donis-Keller et al., 1987), there being about $10^{5}$ RFLPs in the human genome. RFLPs are typed by Southern blotting as shown in Figure 2, the probe being a DNA fragment that spans the polymorphic region (possibly one of the polymorphic fragments, possibly a fragment prepared by cutting the DNA with a different restriction enzyme, or possibly a fragment obtained by PCR) and the presence or absence of the polymorphic site determined from the size(s) of the restriction fragment(s) detected after Southern blotting.

\section{Summary}

Southern blotting is a technique that enables a specific restriction fragment to be detected against a background of many other restriction fragments. It involves transfer of DNA fragments from an electrophoresis gel to a nitrocellulose or nylon membrane in such a way that the DNA banding pattern present in the gel is reproduced on the membrane. Hybridization probing is then used to detect the restriction fragment that is being sought. The basic methodology for Southern blotting has not changed since the original technique was described in 1975, but modifications have been introduced with the aim of speeding up the process and achieving a more efficient transfer. Southern blotting has many applications in molecular biology, including the identification of one or more restriction fragments that contain a gene or other DNA sequence of

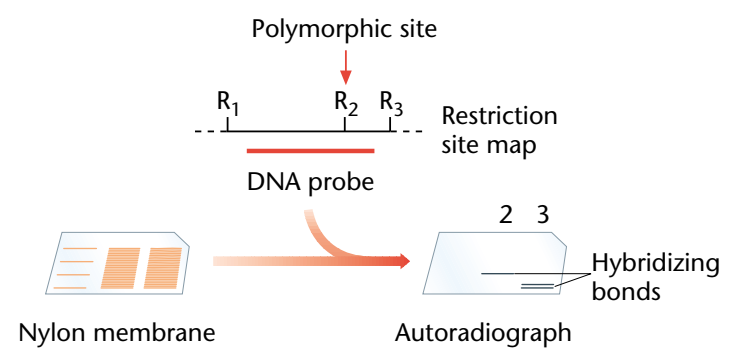

Figure 2 Detection of an RFLP by Southern blotting. In this example, lane 2 contains DNA that lacks the polymorphic restriction site $R_{2}$, and so contains a single restriction fragment that hybridizes with the probe. The DNA in lane 2 contains the polymorphic site and so yields two fragments that hybridize with the probe. Reproduced from Brown (1999) with permission of BIOS Scientific Publishers Ltd, Oxford.

interest, and in the detection of RFLPs used in construction of genomic maps.

\section{References}

Amasino RM (1986) Acceleration of nucleic acid hybridization rate by polyethylene glycol. Analytical Biochemistry 152: 304-307.

Bittner M, Kupferer P and Morris CF (1980) Electrophoretic transfer of proteins and nucleic acids from slab gels to diazobenzyloxymethyl cellulose or nitrocellulose sheets. Analytical Biochemistry 102: 459471.

Brown TA (1999) Genomes. Oxford: BIOS Scientific Publishers.

Donis-Keller H, Green P, Helms C et al. (1987) A genetic map of the human genome. Cell 51: 319-337.

Southern EM (1975) Detection of specific sequences among DNA fragments separated by gel electrophoresis. Journal of Molecular Biology 98: 503-517.

\section{Further Reading}

Ausubel FM, Brent R, Kingston RE et al. (eds) (1993) Current Protocols in Molecular Biology. New York: John Wiley.

Brown TA (1995) Gene Cloning: An Introduction, 3rd edn. London: Chapman \& Hall.

Brown TA (1998) Molecular Biology Labfax. London: Academic Press.

Dyson NJ (1991) Immobilization of nucleic acids and hybridization analysis. In: Brown TA (ed.) Essential Molecular Biology: A Practical Approach, vol. 2, pp. 111-156. Oxford: Oxford University Press.

Sambrook J, Fritsch EF and Maniatis T (1989) Molecular Cloning. A Laboratory Manual. Cold Spring Harbor, NY: Cold Spring Harbor Laboratory.

Watson JD, Gilman M, Witkowski J and Zoller M (1992) Recombinant $D N A$, 2nd edn. New York: WH Freeman. 